Rev. Bras. Saúde Prod. Anim., Salvador, v.16, n.3, p.535-543 jul./set.., $2015 \quad \underline{\text { http://www.rbspa.ufba.br }}$

\title{
Balanço de minerais e desempenho em frangos de corte aos 21 dias de idade alimentados com rações contendo Solanum malacoxylon
}

\author{
Minerals balance and performance of broilers at 21 days of age fed diet containing \\ "Solanum malacoxylon"
}

\author{
VIEITES, Flávio Medeiros ${ }^{1 *}$; ARAÚJO, Gilson Mendes ${ }^{2}$; VALE, Patricia Azevedo \\ Castelo Branco do ${ }^{3}$; SOUZA, Christiane Silva ${ }^{4}$; NÉVOA, Marcelo Lopes ${ }^{5}$; VARGAS \\ JÚNIOR, José Geraldo de ${ }^{6}$; NUNES, Ricardo Vianna ${ }^{7}$; ARRUDA, Nelson Vital \\ Monteiro de ${ }^{8}$
}

\footnotetext{
${ }^{1}$ Universidade Federal de Juiz de Fora, Juiz de Fora, Minas Gerais, Brasil.

${ }^{2}$ Universidade Federal Rural de Pernambuco, Areia, Paraíba, Brasil.

${ }^{3}$ Faculdade de Ciências Sociais Aplicadas, Sinop, Mato Grosso, Brasil.

${ }^{4}$ Universidade Federal de Viçosa, Programa de Pós-Graduação em Bioquímica Aplicada, Viçosa, Minas Gerais, Brasil.

${ }^{5}$ Universidade Federal de Mato Grosso, Programa de Pós-Graduação em Ciência Animal, Cuiabá, Mato Grosso, Brasil.

${ }^{6}$ Universidade Federal do Espírito Santo, Alegre, Espírito Santo, Brasil.

${ }^{7}$ Universidade Estadual do Oeste do Paraná, Marechal Cândido Rondon, Paraná, Brasil.

${ }^{8}$ Universidade Federal de Mato Grosso, Rondonópolis, Mato Grosso, Brasil.

*Endereço para correspondência: fmvieites@yahoo.com.br
}

\section{RESUMO}

Estudou-se o desempenho produtivo $\mathrm{e}$ as quantidades de cálcio $(\mathrm{Ca})$, fósforo $(\mathrm{P})$, magnésio $(\mathrm{Mg})$, potássio $(\mathrm{K})$ e sódio $(\mathrm{Na})$ nas excretas e carcaças de frangos de corte aos 21 dias de idade alimentados com adição de Solanum malacoxylon (SM) nas rações. Adotou-se delineamento inteiramente casualizado, com três tratamentos: $0,0,2,5$ e $5,0 \mathrm{~g}$ de $\mathrm{SM} / \mathrm{kg}$ de ração, sendo cinco repetições e quatro aves por unidade experimental. O uso de 5,0 g de SM/kg de ração reduziu o ganho de peso e piorou a conversão alimentar, embora o consumo não tenha sido influenciado. A inclusão da planta não afetou $(\mathrm{p}>0,05)$ os teores de cálcio, magnésio, potássio e sódio nas excretas e, consequentemente, na absorção desses minerais. A composição mineral das carcaças foi alterada, sendo as concentrações de $\mathrm{Na}, \mathrm{Mg}$ e $\mathrm{K}$, influenciadas. Recomenda-se a adição de até $2,5 \mathrm{~g}$ de $\mathrm{SM} / \mathrm{kg}$ de ração para frangos de corte até 21 dias de idade.

Palavras-chave: 1,25 dihidroxicolecalciferol, carcaça, excretas, retenção mineral

\section{SUMMARY :}

It was studied the performance and the amounts of calcium $(\mathrm{Ca})$, phosphorus $(\mathrm{P})$, magnesium $(\mathrm{Mg})$, potassium $(\mathrm{K})$ and sodium $(\mathrm{Na})$ excreta and carcasses of broilers at 21 days of age fed addition of Solanum malacoxylon (SM) in rations. It was used a completely randomized design with three treatments: $0.0,2.5$ and $5.0 \mathrm{~g}$ of SM/kg diet, five replicates of four birds each. Using $5.0 \mathrm{~g}$ of $\mathrm{SM} / \mathrm{kg}$ diet reduced weight gain, decreased feed conversion, although consumption has not been affected. The inclusion of the plant did not affect $(p>0.05)$ the levels of calcium, magnesium, potassium and sodium in excreta, and consequently the absorption of these minerals. The mineral composition of the carcasses was changed, and the concentrations of $\mathrm{Na}, \mathrm{Mg}$ and $\mathrm{K}$ influenced. It is recommended that the addition to $2.5 \mathrm{~g}$ of $\mathrm{SM} / \mathrm{kg}$ of feed for broilers up to 21 days of age.

Keywords: 1,25 dihydroxycholecalciferol, carcass, excretas, mineral retention 
Rev. Bras. Saúde Prod. Anim., Salvador, v.16, n.3, p.535-543 jul./set.., $2015 \quad \underline{\text { http://www.rbspa.ufba.br }}$

\section{INTRODUÇÃO}

Além da fundamental contribuição do melhoramento genético, o sucesso da avicultura decorre da nutrição. $\mathrm{O}$ ganho de peso médio diário foi de 20 , para mais de $50 \mathrm{~g} / \mathrm{dia}$, e a idade de abate das aves diminuiu de 12 para seis semanas (PONSO et al., 2012). Com a redução da idade de abate, verificou-se um aumento na ocorrência de desordens metabólicas e distúrbios locomotores, que, por sua vez, levam à mobilidade condicionada ou mesmo a claudicação.

$\mathrm{Na}$ criação de frangos de corte, perdas de até $13 \%$ têm sido observadas no processo produtivo, decorrentes das altas densidades de criação das aves, da inadequação mineral, bem como dos níveis de vitamina $\mathrm{D}$ nas dietas (BERTECHINI, 2014). Neste contexto, estudos vêm sendo realizados na avicultura utilizando a vitamina $\mathrm{D}_{3}$, buscando uma melhor relação entre o crescimento e a qualidade do tecido ósseo, de modo a reduzir os problemas locomotores (SOUZA et al., 2013; SOUZA \& VIEITES, 2014; VIEITES et al., 2014). Os metabólitos hidroxilados da vitamina $\mathrm{D}_{3}$ e o 7-dehidrocolesterol já foram identificados em várias plantas, principalmente as pertencentes à família Solanaceae (JÄPELT \& JAKOBSEN, 2013).

A Solanum malacoxylon (SM) ou glaucoplyllum ou glaucum (CHENG et al., 2004; ROVEGNO et al., 2012) é uma planta de habitat palustre encontrada no pantanal de Mato Grosso, sendo um fitógeno rico no componente ativo similar à molécula 1,25dihidroxicolecalciferol $\left(1,25(\mathrm{OH})_{2} \mathrm{D}_{3}\right)$, o calcitriol, fórmula hormonal da vitamina $\mathrm{D}_{3}$ (TOKARNIA et al., 2000). A Solanum malacoxylon contém a vitamina $\mathrm{D}_{3}$ principalmente em suas folhas, mantendo-a mesmo quando secas. Sabe-se que o excesso desta molécula no organismo, por via exógena, produz aumento da concentração de proteína carreadora de cálcio, proporcionando hipercalcemia e hiperfosforemia (MELLO-SILVA et al., 2006).

As avaliações do fornecimento suplementar 1,25-dihidroxivitamina $\mathrm{D}_{3}$ glicosídeo de origem herbal nas rações para frangos de corte ainda são escassas no Brasil; desta maneira, há a necessidade de se obter informações acerca dos possíveis efeitos no desempenho produtivo das aves para que correlações ideais possam ser estabelecidas. Objetivou-se com este estudo, avaliar o desempenho, a composição mineral da carcaça e das excretas de frangos de corte aos 21 dias de idade, alimentados com rações contendo diferentes níveis de inclusão de folhas secas de Solanum malacoxylon.

\section{MATERIAL E MÉTODOS}

O experimento foi realizado na Faculdade de Medicina Veterinária e Zootecnia da Universidade Federal de Mato Grosso (UFMT), Cuiabá-MT. Foram utilizados 60 frangos de corte, machos, da marca comercial $\mathrm{Cobb}^{\circledR}$, alojados com um dia de idade. O delineamento experimental utilizado foi o inteiramente casualizado (DIC), com três tratamentos, cinco repetições e quatro aves por unidade experimental. As aves foram distribuídas uniformemente nas gaiolas no primeiro dia de vida.

As rações foram formuladas à base de milho, farelo de soja e Solanum malacoxylon, nos níveis de 0,$0 ; 2,5$ e $5,0 \mathrm{~g} / \mathrm{kg}$ de ração (Tabela 1 ), seguindo- 
Rev. Bras. Saúde Prod. Anim., Salvador, v.16, n.3, p.535-543 jul./set.., $2015 \quad \underline{\text { http://www.rbspa.ufba.br }}$ ISSN 15199940

se as recomendações de Rostagno et al. (2005).

A Solanum glaucoplyllum apresenta $54,3 \%$ de carboidratos, $24,9 \%$ de proteínas, $4,1 \%$ de água, $17,1 \%$ de minerais (BACHMANN et al., 2013) e glicosídeos de $1,25(\mathrm{OH})_{2} \mathrm{D}_{3} \quad(8,6 \quad \mathrm{a}$ $100 \mu \mathrm{g} / \mathrm{g}$ de folhas secas), entretanto, tal quantidade dependerá das condições ambientais em que a planta foi cultivada, bem como de sua genética. A distribuição molecular dos radicais glicosilados na Solanum é de uma a 12 unidades de hexoses por aglicona (EFSA, 2015).

As aves receberam água e ração à vontade durante todo $\mathrm{o}$ período experimental. As rações foram pesadas e armazenadas em baldes escuros com tampa para posterior distribuição nos comedouros durante o experimento.

Tabela 1. Composição percentual e calculada das rações experimentais

\begin{tabular}{|c|c|c|c|}
\hline Ingrediente $(\%)$ & Tratamento 1 & Tratamento 2 & Tratamento 3 \\
\hline Milho & 60,00 & 59,75 & 59,50 \\
\hline Farelo de Soja & 36,00 & 36,00 & 36,00 \\
\hline Núcleo vitamínico e mineral* & 4,00 & 4,00 & 4,00 \\
\hline Solanum malacoxylon & 0,00 & 0,25 & 0,50 \\
\hline Total $(\%)$ & 100,00 & 100,00 & 100,00 \\
\hline \multicolumn{4}{|c|}{ Composição Calculada } \\
\hline Energia Metabolizável (kcal/kg) & 2861 & 2853 & 2844 \\
\hline Proteína bruta $(\%)$ & 21,27 & 21,25 & 21,23 \\
\hline Cálcio (\%) & 0,981 & 0,981 & 0,981 \\
\hline Fósforo disponível (\%) & 0,415 & 0,415 & 0,414 \\
\hline Fósforo total $(\%)$ & 0,655 & 0,655 & 0,653 \\
\hline Lisina digestível $(\%)$ & 1,044 & 1,043 & 1,042 \\
\hline Metionina + cistina digestível $(\%)$ & 0,757 & 0,756 & 0,756 \\
\hline Treonina digestível $(\%)$ & 0,727 & 0,726 & 0,726 \\
\hline Triptofano digestível (\%) & 0,248 & 0,248 & 0,248 \\
\hline Sódio (\%) & 0,202 & 0,202 & 0,202 \\
\hline
\end{tabular}

*Núcleo Frimix ${ }^{\circledR}$ inicial para frangos de corte: cálcio: $212,0 \mathrm{~g}$; cobre: $3125,0 \mathrm{mg}$; fósforo: $74,7 \mathrm{~g}$; iodo: 32,50mg; manganês: 1906,0mg; pantetonato de cálcio: 237,50mg; selênio: 6,8mg; sódio: 40,0g; zinco: 2281,0mg, ácido nicotínico: 866,0mg; antioxidante: 100,00mg; biotina: 4,0mg; colina: 15,00g; coccidiostático: 3125,0mg; DL-metionina: 42,50g;promotor de crescimento: 1250,0mg; vitamina A: $175000,0 \mathrm{UI} / \mathrm{kg}$; vitamina B1: 44,50mg; vitamina B12: $250,0 \mathrm{mg}$; vitamina B2: 240,00mg; vitamina B6: $86,60 \mathrm{mg}$; vitamina $\mathrm{D}_{3}: 7500,0 \mathrm{UI} / \mathrm{kg}$; vitamina E: $625,0 \mathrm{mg}$; vitamina $\mathrm{K}: 24,50 \mathrm{mg}$.

As aves foram alojadas durante 21 dias, em galpão de alvenaria, com pé direito de três metros de altura, cobertura com laje provida de exaustor e telhas de cimento amianto. Foram utilizadas 15 gaiolas metabólicas com área de $0,24 \mathrm{~m}^{2}$ $(0,60 \mathrm{~m} \times 0,40 \mathrm{~m})$. As médias das temperaturas mínima e máxima registradas durante todo $\mathrm{o}$ período experimental foram de 26,0 e $31,0^{\circ} \mathrm{C}$, respectivamente. A umidade relativa do ar média foi de $69,0 \%$.
O consumo de ração foi calculado pela diferença entre a quantidade de ração fornecida no início e no final do período experimental, levando-se em consideração as sobras das rações. Para determinar o ganho de peso, as aves foram pesadas no inicio e no final da fase. A partir dos dados de consumo de ração e de ganho de peso, calculou-se a conversão alimentar dos animais.

No vigésimo primeiro dia, o experimento foi finalizado, e duas aves, 
com o peso próximo à média do boxe, foram pesadas e abatidas, utilizando a técnica de deslocamento cervical, seguindo os princípios éticos de experimentação animal. Posteriormente, as carcaças das aves foram moídas com penas, cabeças e pés para obtenção de amostras individuais que foram desengorduradas (utilizando éter etílico) e pré-secadas em estufa de ventilação forçada a $55,0^{\circ} \mathrm{C}$ durante 72 horas.

$\mathrm{O}$ ensaio de metabolismo utilizou a metodologia de coleta total de excretas, sendo compreendido por um período de quatro dias de adaptação e três dias de coleta de excretas. Esta foi realizada em cada unidade experimental, uma vez ao dia. As excretas foram acondicionadas em sacos plásticos, devidamente identificados, pesadas e armazenadas em freezer a $-10^{\circ} \mathrm{C}$, para posterior realização das análises laboratoriais. As excretas foram descongeladas e misturadas uniformemente. Depois, foram pré-secadas em estufa de ventilação forçada durante 72 horas a $65^{\circ} \mathrm{C}$. Em seguida, foram moídas e acondicionadas em recipientes plásticos. Procederam-se as análises de matéria seca, cinzas e concentrações de minerais (cálcio $-\mathrm{Ca}$, fósforo $-\mathrm{P}$, sódio $-\mathrm{Na}$, magnésio - $\mathrm{Mg}$ e potássio $-\mathrm{K}$ ) nas amostras (carcaças e excretas), seguindo-se a metodologia de Silva \& Queiroz (2002). A avaliação do conteúdo de $\mathrm{Ca}, \mathrm{Mg}, \mathrm{Na}$ e $\mathrm{K}$ foi realizada por técnica de espectrofotometria de emissão óptica com fonte de plasma indutivamente acoplado (ICP-OES), utilizando espectrômetro simultâneo Perkin Elmer (Optima 3300 DV). Os percentuais de fósforo foram determinados por colorimetria, utilizando o aparelho autoanalisador paramétrico (Alizeé) e Kit da Synermed $^{\mathbb{R}}$.

Os dados obtidos foram submetidos à análise de distribuição normal, pelo critério de informação de KolmogorovSmirnov, análise de variância e, quando significativo, foi realizado o teste de Tukey, ao nível de $5,0 \%$ de probabilidade. Para análise, foi utilizado o software para análise estatística $\mathrm{e}$ genética(SAEG) versão 9.1 (UFV, 2007).

\section{RESULTADOS E DISCUSSÃO}

A inclusão de Solanum malacoxylon influenciou de forma negativa as variáveis de desempenho dos frangos de corte de um a 21 dias de idade (Tabela 2). O uso de 5,0g da planta/kg de ração reduziu o ganho de peso, piorou a conversão alimentar, embora o consumo não tenha sido influenciado.

Tabela 2. Desempenho de frangos de corte de um a 21 dias, alimentados com diferentes níveis de inclusão de Solanum malacoxylon nas rações

\begin{tabular}{lccc}
\hline Solanum malacoxylon & Ganho de peso $(\mathrm{kg})$ & Consumo de ração $(\mathrm{kg})$ & Conversão alimentar \\
\hline $0,0 \mathrm{~g} / \mathrm{kg}$ & $0,820^{\mathrm{A}}$ & $1,160^{\mathrm{A}}$ & $1,414^{\mathrm{A}}$ \\
$2,5 \mathrm{~g} / \mathrm{kg}$ & $0,784^{\mathrm{AB}}$ & $1,154^{\mathrm{A}}$ & $1,473^{\mathrm{AB}}$ \\
$5,0 \mathrm{~g} / \mathrm{kg}$ & $0,705^{\mathrm{B}}$ & $1,058^{\mathrm{A}}$ & $1,505^{\mathrm{B}}$ \\
$\mathrm{CV}(\%)$ & 8,96 & 7,09 & 3,00 \\
\hline
\end{tabular}

*Médias seguidas de mesma letra na coluna não diferem entre si ao nível de 5,0\% de probalibidade pelo teste Duncan.

$\mathrm{CV}=$ coeficiente de varação. 
Rev. Bras. Saúde Prod. Anim., Salvador, v.16, n.3, p.535-543 jul./set.., $2015 \quad \underline{\text { http://www.rbspa.ufba.br }}$ ISSN 15199940

Brito et al. (2010) avaliaram a suplementação de vitamina D (20,0; 37,$5 ; 87,5 ;$ e $137,5 \mu \mathrm{g} / \mathrm{kg}$ de ração) e duas fontes $\left(\mathrm{D}_{3}\right.$ e $\left.25-\mathrm{OHD}_{3}\right)$ isoladas ou associadas, para frangos de corte, e verificaram não haver interação entre as fontes da vitamina $\mathrm{D}$ no desempenho das aves de um a 21 dias. Ao contrário dos resultados deste estudo, os autores mencionados constataram que os níveis de suplementação da vitamina D isolados apresentaram influência positiva no ganho de peso e na conversão alimentar, à medida que se elevaram os níveis da vitamina nas rações na fase inicial. Rao et al. (2006) também verificaram aumento no ganho de peso e melhor conversão alimentar para frangos nesta fase de criação, alimentados com $60 \mu \mathrm{g}$ de vitamina $\mathrm{D}_{3} / \mathrm{kg}$ de ração $(2400 \mathrm{UI} / \mathrm{kg})$, além de níveis reduzidos de cálcio $(0,50 \%)$ e fósforo disponível $(0,25 \%)$ nas rações.

Resultados zootécnicos satisfatórios, em termos de aumento no ganho de peso e melhor conversão alimentar, vêm sendo encontrados quando as aves são suplementadas com a vitamina $\mathrm{D}_{3}$, em suas diferentes formas, em dietas com níveis de cálcio e fósforo disponível reduzidos (CHENG et al., 2004; RAO et al., 2006; SOUZA et al., 2013). Ainda, salienta-se que a fase inicial de crescimento dos frangos de corte é mais susceptível a alterações mediante a suplementação de vitamina $\mathrm{D}$, pois é o período em que ocorre alta taxa de crescimento do tecido esquelético, além da imaturidade do trato digestório digestão e absorção de lipídios e compostos lipossolúveis (GOFF, 2006).

Os resultados observados no presente estudo foram semelhantes aos relatados por Ross et al. (1971), que forneceram Solanum malacoxylon - SM (0,1, 0,5; 1,$0 ; 5,0$ e $10 \%$ de SM nas rações) para galos até três semanas de idade. Os autores descreveram que, ao aumentar o percentual de planta na dieta $(1,0 ; 5,0 \mathrm{e}$ $10 \%$, respectivamente), houve decréscimo no ganho de peso, aumento do cálcio sérico e da mortalidade das aves.

$\mathrm{O}$ calcitriol, ou 1,25- $(\mathrm{OH})_{2} \mathrm{D}_{3}$, induz aumento das concentrações séricas de cálcio em seu principal órgão alvo, o intestino (DiBARTOLA, 2007), e induz a absorção de cálcio principalmente por mecanismo ativo (PEREIRA et al., 2009). Desta forma, aumento no gasto de energia para absorção e transporte do cálcio também pode ter contribuído para a piora no desempenho dos frangos de corte.

A inclusão de Solanum malacoxylon não afetou $(\mathrm{p}>0,05)$ os teores de $\mathrm{Ca}$, $\mathrm{Mg}, \mathrm{K}$ e $\mathrm{Na}$ nas excretas e, consequentemente, na absorção desses minerais (Tabela 3 ).

Tabela 3. Teores de cálcio, fósforo, magnésio, potássio e sódio nas excretas de frangos de corte aos 21 dias de idade, alimentados com Solanum malacoxylon nas rações

\begin{tabular}{lcccc}
\hline \multirow{2}{*}{ Mineral (\%) } & \multicolumn{3}{c}{ Níveis de Solanum malacoxylon } & \multirow{2}{*}{$\mathrm{CV}(\%)$} \\
\cline { 2 - 4 } & $0,0 \mathrm{~g} / \mathrm{kg}$ & $2,5 \mathrm{~g} / \mathrm{kg}$ & $5,0 \mathrm{~g} / \mathrm{kg}$ & 11,70 \\
Cálcio & $11,068^{\mathrm{a}}$ & $11,914^{\mathrm{a}}$ & $11,614^{\mathrm{a}}$ & 8,30 \\
Fósforo & $8,997^{\mathrm{b}}$ & $9,780^{\mathrm{ab}}$ & $10,93^{\mathrm{a}}$ & 13,10 \\
Magnésio & $4,330^{\mathrm{a}}$ & $4,912^{\mathrm{a}}$ & $4,694^{\mathrm{a}}$ & 11,30 \\
Potássio & $18,020^{\mathrm{a}}$ & $17,320^{\mathrm{a}}$ & $17,03^{\mathrm{a}}$ & 18,70 \\
Sódio & $3,558^{\mathrm{a}}$ & $3,690^{\mathrm{a}}$ & $3,306^{\mathrm{a}}$ & \\
\hline
\end{tabular}

*Médias seguidas de mesma letra nas linhas não diferem entre si pelo teste de Tukey ao nível de 5\% de probabilidade.

$\mathrm{CV}=$ coeficinete de variação. 
Rev. Bras. Saúde Prod. Anim., Salvador, v.16, n.3, p.535-543 jul./set.., $2015 \quad \underline{\text { http://www.rbspa.ufba.br }}$ ISSN 15199940

Nas células endoteliais do intestino, $\mathrm{o}$ $1,25(\mathrm{OH})_{2} \mathrm{D}_{3}$ estimula a absorção ativa de cálcio no duodeno, regulada pelo estímulo à expressão de proteínas responsáveis pela captação do cálcio pelos enterócitos, de proteínas envolvidas no transporte intracelular do cálcio (calbindina ou proteína transportadora de cálcio, PTCa) e dos canais de membrana ATP-dependentes para a extrusão do cálcio para o fluido extracelular (CASTRO, 2011; SOUZA \& VIEITES, 2014). Contrariamente ao esperado, não houve aumento de $\mathrm{Ca}$ nas excretas com a adição da planta nas rações, podendo não ter existido interferência na absorção de Ca nas aves devido à saturação da proteína carreadora de $\mathrm{Ca}$, que é ativada pela vitamina $\mathrm{D}$.

O acréscimo de Solanum malacoxylon afetou o teor de fósforo $(\mathrm{P})$, sendo que o maior valor excretado foi verificado em $5,0 \mathrm{~g}$ de $\mathrm{SM} / \mathrm{kg}$ de ração. Igualmente ao $\mathrm{Ca}$, o processo de absorção do $\mathrm{P}$ é estimulado pela vitamina $D$ na sua forma mais ativa, além de ser dependente de sódio.

Guyton \& Hall (2006) relataram que a concentração de $\mathrm{P}$ inorgânico $\left(\mathrm{P}_{\mathrm{i}}\right)$ é reduzida por efeitos diretos sobre a excreção renal, sendo o hormônio paratormônio $(\mathrm{PTH})$ o responsável pela excreção elevada de $\mathrm{P}_{\mathrm{i}}$. Os autores afirmaram que, na tentativa de manter o equilíbrio dos níveis séricos de $\mathrm{Ca}$ e de $\mathrm{P}$ em baixas concentrações de $\mathrm{P}_{\mathrm{i}}$ no sangue, ocorre elevação do nível sérico da concentração de $\mathrm{Ca}^{++}$no organismo, através da remoção óssea. Com a inclusão da planta nas dietas, as aves aumentaram a excreção do $\mathrm{P}$, provavelmente, na tentativa de manter a homeostase.

A composição mineral das carcaças foi alterada $(\mathrm{p}<0,05)$ pela adição de Solanum malacoxylon nas rações, de modo que as concentrações de $\mathrm{Na}, \mathrm{Mg}$ e $\mathrm{K}$ foram influenciadas (Tabela 4).

Tabela 4. Teores de cálcio, fósforo, magnésio, potássio e sódio nas carcaças de frangos de corte aos 21 dias de idade, alimentados com Solanum malacoxylon nas rações

\begin{tabular}{lcccc}
\hline \multirow{2}{*}{ Mineral (\%) } & \multicolumn{3}{c}{ Níveis de Solanum malacoxylon } & \multirow{2}{*}{ CV (\%) } \\
\cline { 2 - 4 } & $0,0 \mathrm{~g} / \mathrm{kg}$ & $2,5 \mathrm{~g} / \mathrm{kg}$ & $5,0 \mathrm{~g} / \mathrm{kg}$ & \\
\hline Cálcio & $1,846^{\mathrm{a}}$ & $1,704^{\mathrm{a}}$ & $1,622^{\mathrm{a}}$ & 14,70 \\
Fósforo & $2,280^{\mathrm{a}}$ & $2,378^{\mathrm{a}}$ & $2,360^{\mathrm{a}}$ & 14,30 \\
Magnésio & $0,155^{\mathrm{a}}$ & $0,107^{\mathrm{b}}$ & $0,117^{\mathrm{ab}}$ & 15,80 \\
Potássio & $0,536^{\mathrm{a}}$ & $0,456^{\mathrm{b}}$ & $0,422^{\mathrm{b}}$ & 9,60 \\
Sódio & $0,372^{\mathrm{a}}$ & $0,298^{\mathrm{b}}$ & $0,264^{\mathrm{b}}$ & 14,00 \\
\hline
\end{tabular}

*Médias seguidas de mesma letra nas linhas não diferem entre si pelo teste Tukey ao nível de $5 \%$ de probabilidade.

$\mathrm{CV}=$ coeficiente de variação.

A diminuição dos teores de $\mathrm{Na}, \mathrm{Mg}$ e $\mathrm{K}$ na carcaça dos frangos que receberam 2,5 e $5,0 \mathrm{~g} / \mathrm{kg}$ de Solanum malacoxylon nas rações aos 21 dias de idade pode estar relacionada ao possível estresse por calor ao final do período experimental. Aves em alcalose respiratória eliminam mais prótons
$\left(\mathrm{Na}^{+}, \mathrm{Mg}^{++}\right.$e $\left.\mathrm{K}^{+}\right)$por via renal na tentativa de manter o equilíbrio ácidobásico do organismo (VIEITES et al., 2004; VIEITES et al., 2009). Sugere-se que frangos de corte alimentados com rações suplementadas com $1,25(\mathrm{OH})_{2} \mathrm{D}_{3}$ mobilizam $\mathrm{Na}^{+}, \mathrm{Mg}^{++}$e $\mathrm{K}^{+}$, quando criados em altas temperaturas. É 
Rev. Bras. Saúde Prod. Anim., Salvador, v.16, n.3, p.535-543 jul./set.., $2015 \quad$ http://www.rbspa.ufba.br ISSN 15199940

provável que essa descompensação tenha ocorrido na última semana da pesquisa, fase em que as aves exigiam uma temperatura inferior à ocorrida no local. O manual $\mathrm{Cobb}^{\circledR}$ recomenda $26^{\circ} \mathrm{C}$ como temperatura ideal para frangos de corte de 17-20 dias de idade, para uma umidade relativa do ar de $70 \%$ (COBBVANTRESS, 2009). Durante o experimento, a temperatura média máxima registrada foi de $31^{\circ} \mathrm{C}$, e a umidade relativa do ar foi de $69 \%$.

Recomenda-se adicionar até $2,5 \mathrm{~g}$ de Solanum malacoxylon (SM)/kg de ração para frangos de corte até 21 dias de idade, uma vez que, até tal nível, não houve o comprometimento das variáveis produtivas das aves.

As quantidades de $\mathrm{Na}, \mathrm{Mg}$ e $\mathrm{K}$ nas carcaças foram reduzidas, à medida que a planta foi incluída nas rações.

Em relação às excretas, apenas 0 fósforo foi influenciado, de modo que a eliminação desse elemento aumentou com o acréscimo de SM nas rações.

\section{AGRADECIMENTOS}

Ao Conselho Nacional de Desenvolvimento Científico e Tecnológico (CNPq), pela concessão do financiamento do projeto de pesquisa; à Universidade Federal de Mato Grosso, campus Cuiabá, pela infraestrutura fornecida e à fazenda Piuval por permitir a coleta do material para experimento (Solanum malacoxylon).

\section{REFERÊNCIAS}

BACHMANN, H.; OFFORD-CAVIN, E.; PHOTHIRATH, P.; HORCAJADA, M.; ROMEIS, P.; MATHIS, G.A. 1,25dyhroxyvitamin $\mathrm{D}_{3}$-glycoside of herbal origin exhibits delayed release pharmacokinetics when compared to its synthetic counterpart. Journal of
Steroid Biochemistry and Molecular

Biology, v.136, p.333-336, 2013.

BERTECHINI, A.G. Exigências de minerais para aves. In: SAKOMURA, N.K.; SILVA, J.H.V.; COSTA, F.G.P.; FERNANDES, J.B.K.; HAUSCHILD, L. Nutrição de não ruminantes. Jaboticabal,SP: FUNEP, 2014. 678p.

BRITO, J.A.G.; BETERCHINI, A.G.; FASSANI, E.J.; RODRIGUES, P.B.; LIMA, E.M.C.; MENEGHETTI, C. Efeito da vitamina $\mathrm{D}_{3}$ e 25-hidroxicolecalciferol sobre o desempenho, o rendimento de carcaça e a morfologia intestinal de frangos de corte. Revista Brasileira de Zootecnia, v.39, p.26562663, 2010.

CASTRO, L.C.G. O sistema endocrinológico vitamina D. Arquivos Brasileiros de Endocrinologia \& Metabologia, v.55, p.566-575, 2011.

CHENG, Y.H.; GOFF, J.P.; SELL, J.L.; DALLORSO, M.E.; GIL, S.;

PAWLAK, S.E.; HORST, R.L.Utilizing Solanum glaucophyllum alone or with phytase to improve phosphorus utilization in broilers. Poultry Science, v.83, p.406-413, 2004.

COBB-VANTRESS Inc.Manual de manejo de frangos de corte. ArkansasUS: S.I, 2009. 70p.

DiBARTOLA, S.P. Anormalidades de fluidos, eletrólitos e equilíbrio ácidobásico na clínica de pequenos animais. São Paulo-SP: Roca, 2007.

EUROPEAN FOOD SAFETY

AUTHORITY - EFSA. Scientific Opinion on the safety of Solanum glaucophyllum standardised leaves as feed material. EFSA Journal, v.13, n.1, p.3967, 2015. 
Rev. Bras. Saúde Prod. Anim., Salvador, v.16, n.3, p.535-543 jul./set.., $2015 \quad$ http://www.rbspa.ufba.br ISSN 15199940

GOFF, J.P. Distúrbios do metabolismo dos carboidratos e da gordura. In: REECE, W.O. DUKES. Fisiologia dos animais domésticos. 12.ed. Rio de Janeiro: Guanabara Koogan, 2006. 926p.

GUYTON, A.C.;HALL, J.E. Tratado de fisiologia médica.11.ed. Rio de Janeiro: Elsevier, 2006.

JÄPELT, R.B.; JAKOBSEN, J. Vitamin $D$ in plants: a review of occurrence, analysis, and biosynthesis. Frontiers in Plant Science, v.4, 2013. DOI: 10.3389/fpls.2013.00136.

MELLO-SILVA, C.C.; LIMA, M.; PINHEIRO, J. BEZERRA, J.C.B; RODRIGUES, M.LA. Alterações fisiológicas em Biomphalaria glabrata tratadas com extrato bruto de Solanum malacoxylon. Ciência Animal, v.16, n.2, p.61-70, 2006.

PEREIRA, G.A.P.; GENARO, P.S.; PINHEIRO, M.M.; SZEJNFELD, V.L.; MARTINI, L.A. Dietary calcium strategies to optimize intake. Revista

Brasileira de Reumatologia, v.49, n.2, p.172-180, 2009.

PONSO, R.; FARIA, D.E.; ALBUQUERQUE, R.; PAZ, I.C.L.A.; ARTONI, S.M.B.; SANTOS, A.L.; SAVIANI, G.; ARAÚJO, C.M.M. Avaliação do desenvolvimento da discondroplasia tibial em frangos de corte submetidos à dieta com 25-hidroxicolecalciferol.

\section{Brazilian Journal of Veterinary}

Research and Animal Science, v.49, n.2, p.153-161, 2012.

RAO, S.V.R.; RAJU, M.V.L.N.; PANDA, A.K.; SUNDER, G.S.; SHARMA, R.P. Effect of high concentrations of cholecalciferol on growth, bone mineralization, and mineral retention in broiler chicks fed suboptimal concentrations of calcium and non phytate phosphorus. Journal Applied Poultry Research, v.15, p.493-501, 2006.

ROSTAGNO, H.S.; ALBINO, L.F.T.; DONZELE, J. L.; GOMES, P.C. OLIVEIRA, R. F.; LOPES, D.C.; FERREIRA, A.S.; BARRETO, S.L.T. Tabelas brasileiras para suínos e aves: composição de alimentos e exigências nutricionais. 2.ed. Viçosa, MG: Universidade Federal de Viçosa, 2005. 186p.

ROSS, E.; SIMPSON,C.F.; ROWLAND, L.O.; HARMS, R.H. Toxicity of Solanum sodomaeum and Solanum malacoxylonto chicks. Poultry Science, v.50, n.3, p.870-873, 1971.

ROVEGNO, S.; MICCOLI, F.; DALLORSO, M.; IGLESIAS, B.; AZCONA, J. Calciotropic principle of Solanum glaucophyllum in broiler chickens.International Journal of Poultry Science, v.11, n.4, p.298-301, 2012.

SILVA, D.J.; QUEIROZ, A.C. Análise de alimentos: métodos químicos e biológicos.3.ed. Viçosa,MG: Universidade Federal de Viçosa, 2002. $235 p$.

SOUZA, C.S.; VIEITES, F.M.; VASCONCELLOS, C.H.F.; CALDERANO, A.A.; NUNES, R.V.; FERREIRA, C.M.; PEREIRA, T.V.S.; MORAES, G.H.K. Suplemento de 1,25 dihidroxicolecalciferol e redução de cálcio e fósforo disponível para frangos de corte. Arquivo Brasileiro de Medicina Veterinária e Zootecnia, v.65, n.2, p.519-525, 2013. 
SOUZA, C.S.; VIEITES, F.M. Vitamin $\mathrm{D}_{3}$ e seus metabólitos para frangos de corte. Archivos de Zootecnia, v.63, p.11-24, 2014.

UNIVERSIDADE FEDERAL DE VIÇOSA - UFV. Sistema de Análise Estatística e Genética - SAEG. Versão 9.1. Viçosa, MG, 2007.

VIEITES, F.M.; ARAÚJO, G.M.; CARAMORI JR, J.G.; VARGAS JR, J.G. Variação catiônica na dieta sobre o peso absoluto, rendimento de carcaça e cortes nobres de frangos de corte na região sul de Mato Grosso. Revista Brasileira de Saúde e Produção Animal [online], v.10, n.3, p.723-730, 2009.

VIEITES, F.M.; MORAES, G.H.K.; ALBINO,L.F.T.; ROSTAGNO, H.S.; RODRIGUES, A.C.; SILVA, F.A.; ATENCIO, A.Balanço eletrolítico e níveis de proteína bruta sobre parâmetros sanguíneos e ósseos de frangos de corte aos 21 diasde idade.

Revista Brasileira de Zootecnia,v.33, n.6, p.1520-1530, 2004.

VIEITES, F.M.; NALON, R.P.; SANTOS, A.L.; BRANCO, P.A.C.; SOUZA, C.S.; NUNES, R.V.; CALDERANO, A.A.; ARRUDA, N.V.M. Desempenho, rendimento de carcaça e cortes nobres de frangosde corte alimentados com rações suplementadas com Solanum glaucophyllum. Semina: Ciências Agrárias, v.35, n.3, p.1617-1626, 2014.

TOKARNIA, C.H.; DOBEREINER, J.; PEIXOTO, P.V. Plantas tóxicas do

Brasil. Rio de Janeiro: Helianthus, 2000. 310p.

Data de recebimento: 27/02/2014

Data de aprovação: 07/09/2015 